\title{
EVALUACIÓN IN VITRO DEL TAMO DE ARROZ, DEGRADADO POR PLEUROTUS OSTREATUS PARA LA ALIMENTACIÓN EN RUMIANTES
}

\section{EVALUATION IN VITRO OF RICE STRAW, DEGRADADED BY PLEUROTUS OSTREATUS, AS A RUMINANT FEED}

\author{
*Tapie C. William A. y ${ }^{1}$ Sánchez G. Hugo'. \\ 1 Zootecnista MSc. Profesor asociado Universidad Nacional de Colombia Sede Palmira \\ Departamento de Ciencia Animal. Correo electrónico: *watapiec@unal.edu.co ; \\ hsanchezgu@unal.edu.co.Colombia.
}

Recibido 21 de Octubre 2015; aceptado 30 de Marzo de 2016

RESUMEN

El tamo de arroz es un material altamente lignificado que puede ser usado alternativamente como alimento para rumiantes; sin embargo la despolimerización de los hidratos de carbono en este material celulósico se ve obstaculizada por el alto contenido de lignina, por tanto se plantea como objetivo hacer un tratamiento al tamo de arroz con Pleurotus ostreatus y evaluar su uso potencial en la alimentación de rumiantes, mediante la técnica de producción de gas in vitro y composición química; se encontró un importante efecto a los 20 días de tratamiento con el hongo sobre la calidad nutricional del tamo, reflejada en el aumento de proteína cruda (PC) hasta un 37 \% más, así como en la reducción del 
contenido de lignina (LDA) y fibra detergente neutra (FDN) de $54 \%$ y $27 \%$ respectivamente. Finalmente la modificación de estos compuestos por el hongo, se vio manifestado en un 40 \% más de producción de gas frente al testigo. De esta manera el tratamiento del tamo de arroz con el hongo lignolitico, se constituye en una alternativa viable para mejorar el valor nutricional de este tipo de subproductos y poder aprovechar mejor este material, especialmente en época de escases forrajera.

Palabras clave: fibra, hongo, lignina, subproducto.

\section{ABSTRACT}

Rice straw is a material highly lignified that can be used as ruminant feed alternative; however, the carbohydrates depolymerization of this cellulosic material is hindered by its high lignin content. Therefore, the objective of this study was to treat rice straw with Pleurotus ostreatus and evaluate its potential as a ruminant feed through the in vitro gas production and chemical composition technique. After 20 days of treatment, an important effect on the nutritional quality of the rice straw was found, there was an increase in the raw protein up to $37 \%$, also there was a reduction on the lignin content and on the neutral detergent fiber (NDF) of 54 and $27 \%$, respectively. Finally, the modification of these compounds by $P$. ostreatus was reflected by $40 \%$ more gas production than the control. The treatment of rice straw with this ligninolytic fungi can be incorporated as a viable alternative to improve the nutritional value of these type of byproducts and take advantage of this material, especially when there is a foliage shortage.

Key Words: fiber, fungi, lignin, byproduct. 


\section{INTRODUCCIÓN}

El material celulósico como la paja de arroz es una biomasa abundante (Abdel-Hamid et al., 2013; FAO, 2012), puede proporcionar una fuente alternativa de alimento para ganado rumiante, debido a la capacidad de éstos para consumir y nutrirse de subproductos con alto contenido de fibra (Montañez et al., 2004; FAO, 2012; AbdelHamid et al., 2013). Sin embargo, el alto contenido de lignina y bajo contenido de proteína de estos residuos minimiza su aprovechamiento al reducir significativamente su digestibilidad (Hungate, 1988; Peláez, 2010; Akin et al., 1993), ya que la despolimerización de los hidratos de carbono en este material celulósico se ve obstaculizada por el alto contenido de lignina, a causa de que esta es resistente a la degradación química y biológica por su compleja estructura química (Abdel-Hamid et al., 2013).

Durante la cosecha del arroz; una de las prácticas más frecuente realizada por los agricultores es la de eliminar la paja, la cual es quemada en el campo debido a la dificultad y elevado costo de su retirada, nulo aprovechamiento (Abril et al., 2009) y baja digestibilidad este último a causa de su composición nutricional con valores de $82 \%$ de FDN (Van Soest, 2006), 90 \% de materia seca (MS), $44 \%$ de nutrientes digestibles totales (TDN), $4.3 \%$ de (PC), $0.2 \%$ de calcio (Ca) y $0.08 \%$ de fosforo (P) (Drake, 2002).

Con el propósito de mejorar las condiciones de este subproducto, se han utilizado métodos químicos, físicos y biológicos para producir la deslignificación o ruptura del complejo carbohidrato lignina y favorecer así el acceso de los microorganismos ruminales a los carbohidratos estructurales, mejorando su digestibilidad (Montañez et al., 2004: Díaz, et al., 2014). En los cuales los procesos biológicos han sido considerados como una alternativa a la generación de bienes y servicios debido a la forma de transformar los residuos en productos útiles (Ferreira, 2009). Dentro de este contexto los hongos lignoceluloliticos como Pleurotus spp, juegan un papel importante en el proceso de deslignificación formando mejores productos de interés para la alimentación animal (Barros, 2009). Este tipo de tratamiento tiene un gran potencial para mejorar la digestibilidad de la paja de arroz, pues estos hongos tienen la capacidad de disminuir los componentes de la pared celular, por medio de la producción de enzimas extracelulares como las peroxidasas y lacasas, ya que estas enzimas fúngicas pueden oxidar compuestos fenólicos como la lignina (Moreira, 2006; Pompeu, 2010; Higuchi, 2004), las cuales 
han llamado la atención por sus valiosas aplicaciones biotecnológicas especialmente en el pretratamiento de la biomasa lignocelulósica (Abdel-Hamid et al., 2013; Moreira, 2006; Cohen, 2002; Eggen, 2000); de este modo se puede aumentar la digestibilidad de los subproductos agrícolas fibrosos, como la paja de arroz (Peláez, 2010; Akin et al., 1993), lo que permite la revalorización de los mismos, además debido a su alta disponibilidad y de ser aprovechado de la mejor manera, sería una alternativa de alimentación para el ganado

\section{MATERIALES Y MÉTODOS}

\section{Reproducción y almacenamiento de $P$. ostreatus}

previamente humedecido $(65 \pm 5 \%)$ y esterilizado.

Se seleccionó una cepa del hongo, $P$. ostreatus proveniente del laboratorio de insumos biológicos FUNGICOL S.A.S. Palmira Valle del Cauca. Colombia. La multiplicación del hongo se realizó en un medio de Potato Dextrose Agar (PDA), de acuerdo a la metodología propuesta por Ferreira (2009). En 1 litro de agua se adiciono $30 \mathrm{~g}$ de extracto de malta, $3 \mathrm{~g}$ de peptona de harina de soja y $15 \mathrm{~g}$ de agar, se llevó al autoclave a $121{ }^{\circ} \mathrm{C} / 15$ minutos, seguidamente se pasó a cajas de Petri con $10 \mathrm{ml}$ del medio y se incubo por 7 días a 30 $\pm 2 \stackrel{\circ}{ } \mathrm{C}$. Luego para su almacenamiento y posterior uso, se inoculó 3 cajas de Petri en bolsas de polipropileno con $500 \mathrm{~g}$ de trigo

\section{Cultivo de $\boldsymbol{P}$. ostreatus en el tamo de arroz}

El tamo, fue reducido de tamaño ( $3 \mathrm{a} 4 \mathrm{~cm}$ ), humedecido (65 $\pm 5 \%$ ) y esterilizado (15 lb, $\left.121^{\circ} \mathrm{C} / 1 \mathrm{~h}\right)$. seguidamente se inoculó el tamo con el hongo dispuesto en el trigo, $250 \mathrm{~g}$ en $5 \mathrm{~kg}$ de tamo de arroz y se dejó que este colonice por un periodo de 20 días en recipientes sin presencia de oxígeno. Transcurrido este tiempo, al tamo de arroz usado como sustrato para el cultivo del hongo y al tamo control, se determinó el contenido de: MS y Cenizas (AOAC, 1990); PC (Kjeldahl, 1983); FDN y LDA (ANKOM) y producción de gas in vitro (Menke y Steingass, 1988). 
@ @iMENTECH CIENCIA Y TECNOLOGÍA ALIMENTARIA ISSN 1692-7125. Volumen 14 No. 1, p. 5 -16, año 2016 Facultad de Ingenierías y Arquitectura

Universidad de Pamplona

\section{Diseño experimental}

Los tratamientos fueron: 1 Tamo de arroz tratado con $P$. ostreatus; 2 tamo control (sin tratamiento). El análisis estadístico se realizo bajo un modelo completamente al azar con 3 repeticiones.

Modelo estadístico:

$\mathbf{Y}_{i j}=\mu+T_{i}+E_{i j}$

$\mathbf{Y}_{\mathrm{ij}}=\mathrm{FDN}$, LDA, MS, PC y cenizas

$\mu=$ Promedio

$\mathbf{T}_{\mathbf{i}}=(\mathbf{i}=1,2)$

$\mathbf{E}_{\mathrm{ij}}=$ Error experimental

\section{Evaluación del grado de deslignificación}

El grado de deslignificación, se estimó de acuerdo a la ecuación (1). El término lignina inicial y final se refiere a la concentración (\%) antes y después del proceso de degradación del tamo de arroz por parte del hongo (Rangel, 2012).

\section{(1) Grado de des lignificación}

\section{(lignina inicial - lignina final) * 100}

\section{Lignina inicial}

Las características morfológicas (cualitativo) de la pared celular del tamo de arroz antes y después de ser sometido al proceso de degradación por $P$. ostreatus, se observaron mediante la técnica de microscopia electrónica de barrido.

Equipo: Microscopio Electrónico de Barrido, Marca: FEI, Modelo: Quanta 200 Metalizador. Esto se realizó en el Laboratorio de Microscopía Electrónica de Barrido de la Universidad Nacional de Colombia. Bogotá.

\section{Producción de gas in vitro con la técnica de fermentación en Jeringas}

El estudio de la cinética de degradación del tamo de arroz a través del volumen de gas producido durante el proceso fermentativo a diferentes tiempos $(2,5,8,12,16,20,24,48$ y 72horas), se realizó de acuerdo a la metodología de Menke y Steingass (1988). Los animales donantes del líquido ruminal fueron dos machos cebuinos con un peso promedio de $900 \mathrm{~kg}$, en pastoreo con pasto estrella (Cynodon plectostachium), suplementados con torta de soya y sal mineralizada. Para estudiar la dinámica de producción de gas se utilizó el modelo propuesto por France et al (1993).

$$
\mathbf{Y}=\mathbf{A}\left[\mathbf{1}-\mathbf{e}^{(-\mathbf{b}(\mathbf{t}-\mathbf{L}))-\mathbf{c}(\sqrt{\mathbf{t}}-\sqrt{\mathbf{L}})}\right]
$$

$\mathrm{Y}=$ Volumen de gas en el tiempo (ml)

$A=$ Volumen de gas acumulado proveniente de la fermentación del sustrato (asíntota)

$\mathrm{b}=$ Tasa constante de producción de gas $(\mathrm{ml} / \mathrm{h})$

$\mathrm{t}=$ Tiempo de incubación (h) 
$\mathrm{C}=$ tasa constante de producción de gases

$\mathrm{L}=$ tiempo de retraso $\mathrm{Lag} / \mathrm{h}$

del material potencialmente degradable.

\section{RESULTADOS Y DISCUSIÓN}

\section{Degradación del tamo de arroz por $\boldsymbol{P}$. ostreatus.}

Inicialmente se encontró que el tamo de arroz-control, tuvo un promedio de $13 \pm$ $0.8 \%$ de lignina (tabla 1 ), que al ser tratado con $P$. ostreatus por un periodo de 20 días, se redujo a $6 \pm 1 \%$ en promedio $(\mathrm{p}<0.01)$, ya que este hongo tienen la capacidad de disminuir los componentes de la pared celular debido a que sintetiza enzimas fibrolíticas (Peláez, 2010).

Tabla 1. Prueba de Duncan, para la composición química del tamo de arroz tratado con $P$. ostreatus y $\sin \operatorname{tratar}(\mathrm{n}=3)$.

\begin{tabular}{ccc}
\hline $\begin{array}{l}\text { Componente } \\
(\%)\end{array}$ & $\begin{array}{l}\text { Tamo de } \\
\text { arroz- } \\
\text { control }\end{array}$ & $\begin{array}{l}\text { Tamo de } \\
\text { arroz } \\
\text { tratado }\end{array}$ \\
\hline MS & $92 \pm 0.3^{\mathrm{a}}$ & $92 \pm 0.2^{\mathrm{a}}$ \\
Cenizas & $13 \pm 0.2^{\mathrm{b}}$ & $15 \pm 0.1^{\mathrm{a}}$ \\
PC & $7 \pm 0.5^{\mathrm{b}}$ & $11 \pm 0.3^{\mathrm{a}}$ \\
FDN & $78 \pm 0.5^{\mathrm{a}}$ & $57 \pm 1.1^{\mathrm{b}}$ \\
LDA & $13 \pm 0.8^{\mathrm{a}}$ & $6 \pm 1.1^{\mathrm{b}}$ \\
\hline \multicolumn{3}{c}{ ab valores con distinta letra en la fila difieren } \\
\multicolumn{3}{c}{ estadísticamente $(\mathrm{p}<0.01)$}
\end{tabular}

La mayoría de los componentes estudiados exceptuado la MS presentaron diferencias altamente significativas $\quad(P<0.01)$. Resultados similares son reportados al evaluar el efecto de la paja de trigo tratada con Pleurotus florida, encontrando que el contenido de MS en la paja tratada y sin tratar fue similar, a diferencia de los contenidos de FDN, PC, cenizas y LDA (Montañez et al., 2004).

La FDN determinante en la alimentación de rumiantes, disminuyo en el tamo tratado $(p<0.01)$ equivalente a un $27 \%$ en comparación con el control (tabla 1), por lo que el hongo pudo haber utilizado parte de estos componentes (celulosa hemicelulosa, lignina) para su crecimiento. Akinfemi y Ogunwole (2012), encontraron que el tratamiento del tamo de arroz con un hongo de este mismo género, hace que los componentes de la pared celular disminuyan, mostrando diferencias estadísticas significativas frente al control. Este efecto es una ventaja relevante, ya que la FDN está directamente relacionada con la digestibilidad de la materia seca. La cual 
@ @iMENTECH CIENCIA Y TECNOLOGÍA ALIMENTARIA ISSN 1692-7125. Volumen 14 No. 1, p. 5 -16, año 2016 Facultad de Ingenierías y Arquitectura

Universidad de Pamplona

para ganado de leche debe encontrarse en los forrajes entre 35 - $45 \%$ (DairyNZ, 2008).

El contenido de PC aumento del $7 \%$ al $11 \%$ en el tamo tratado. La biomasa de $P$. ostreatus rica en proteína presente en el tamo de arroz explica este importante hallazgo, lo que explicaría también el aumento de cenizas (Mushrooms, 2007). En estudios relacionados, se ha encontrado que al ser tratado el tamo de arroz con Pleurotus aumenta el contenido de PC de 4.69 a 7.69 $\%$ (Akinfemi y Ogunwole, 2012).

En términos porcentuales, el tratamiento con el hongo indicó tener una eficiencia de deslignificación del 54 \% (Ecuación 2). En estudios referentes, Rangel (2012) evaluó el efecto del complejo enzimático sobre la degradación de $P$. maximum y obtuvo un grado de deslignificación del 25.5 \%. Así mismo Taniguchi et al., (2005) en pre tratamiento de cascarilla de arroz con $P$. ostreatus, encontraron un grado de des lignificación del $41 \%$.

$$
\begin{aligned}
& \text { Grado de deslignificación } \\
& \qquad=\frac{(13-6)}{13} * 100=54 \%
\end{aligned}
$$
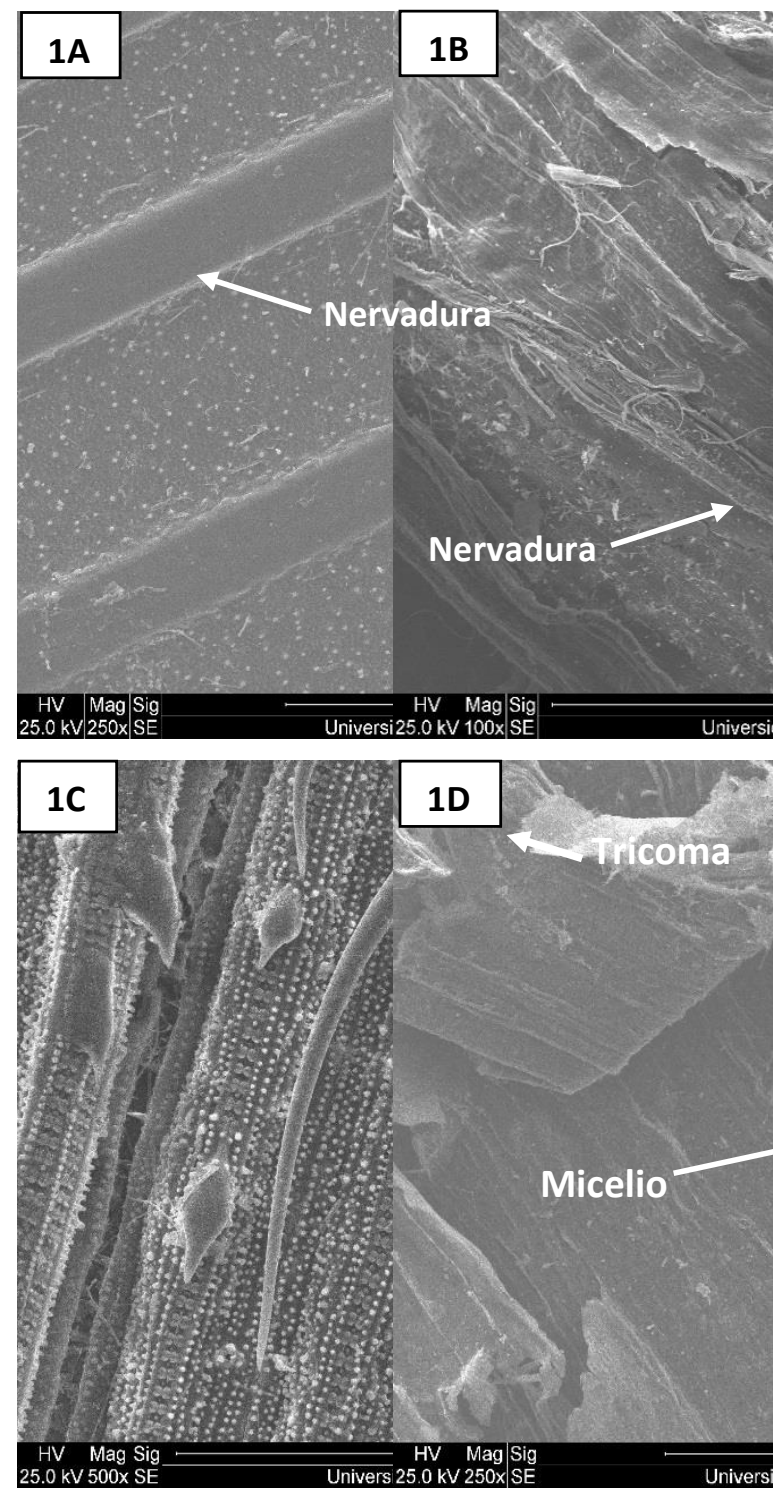

Figura 1. Microscopía electrónica de barrido, del tamo de arroz antes y después de tratamiento con $P$. ostreatus. $1 \mathrm{~A}$. Nervaduras de la hoja; 1B. Nervaduras de la hoja tratada 1C. Tricomas; 1D. Colonización del micelio en la hoja.

La microscopía electrónica de barrido (análisis cualitativo), muestra de forma general la biomasa modificada después de la colonización del hongo durante 20 días; en la 
@ @iMENTECH CIENCIA Y TECNOLOGÍA ALIMENTARIA ISSN 1692-7125. Volumen 14 No. 1, p. 5 -16, año 2016 Facultad de Ingenierías y Arquitectura Universidad de Pamplona

figura " $1 \mathrm{~A}$ " se puede apreciar las nervaduras paralelas de la hoja, quienes sufrieron una degradación por parte del hongo, y su contraste "1B"; así mismo en la figura " $1 \mathrm{C}$ " se observan los tricomas de la superficie de la hoja cuyas características de estos es que presentan paredes celulósicas, recubiertas de cutícula, o paredes secundarias lignificadas (González y Arbo, 2013), la cual pudo ser degradada por el complejo enzimático de $P$. ostreatus al ser colonizado como se muestra en la figura "1D" y de este modo contribuir a la disminución de LDA. Por último el cambio de las características morfológicas después del tratamiento es evidente, con lo que se puede soportar el porcentaje de deslignificación tan eficiente conseguido (54\%). Estudios han demostrado que la variación en la textura observados bajo microscopia electrónica de material vegetal expuesto a hongos lignoliticos, facilita la penetración microbiana ruminal al tejido lignificado y en consecuencia aumenta la digestibilidad del mismo (Karunanandaa et al., 995)

\section{Producción de gas in vitro}

En la dinámica de producción de gas representada bajo el modelo propuesto por France et al., (1993), se encontró que todos los parámetros fueron significativos $(\mathrm{p}<0.05)$. La producción de gas acumulado fue de 46.3 y $28.1 \mathrm{ml}$, tasa de producción de gas de 0.01 y $0.043 \mathrm{ml} / \mathrm{h}$, lag de 0.003 y 2.03 (h), para el tamo tratado y control respectivamente (figura 2). Esto debido a que el contenido de LDA y FDN en el tamo tratado fue menor (tabla 1). Ya que la despolimerización de los hidratos de carbono en la biomasa lignocelulósica se ve obstaculizada por la lignina que es resistente a la degradación química y biológica (Abdel-Hamid et al., 2013).

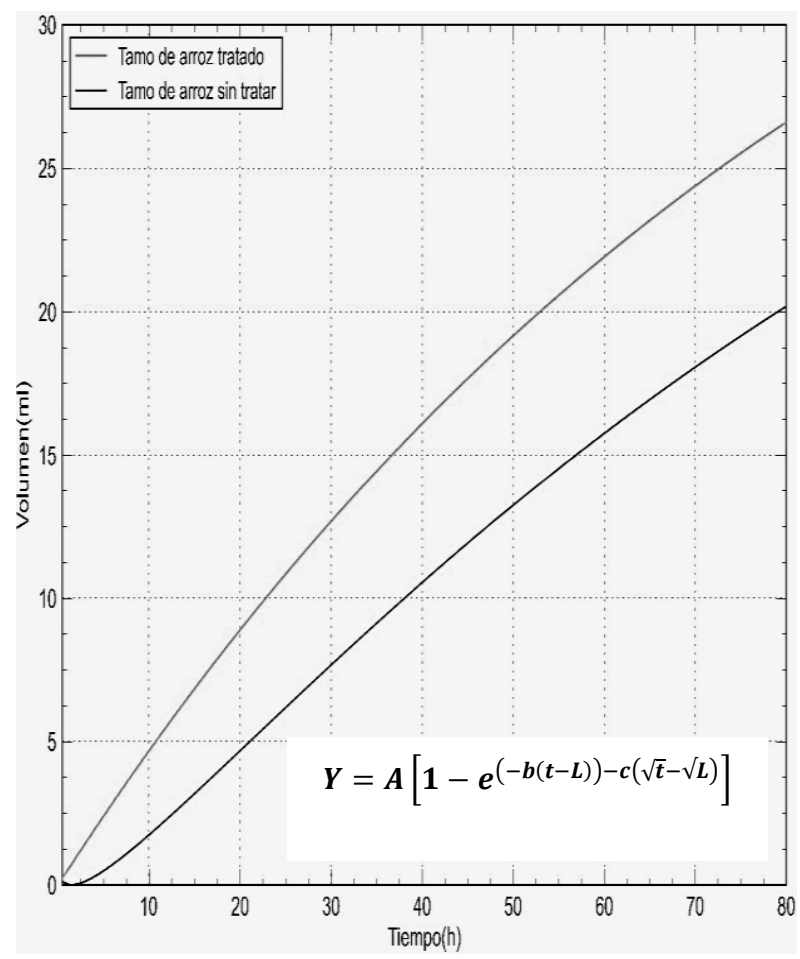

Figura 2. Cinética de producción de gas de tamo de arroz tratado con P. ostreatus y control.

Estudios relacionados, han demostraron que el tratamiento con las enzimas ligninolíticas de Pleurotus aumenta la producción neta de gas y la digestibilidad de la FDN de las pajas de los residuos agrícolas. El tratamiento con $P$. ostreatus ayuda a la disminución de LDA, 
@LIMENTECH CIENCIA Y TECNOLOGÍA ALIMENTARIA ISSN 1692-7125. Volumen 14 No. 1, p. 5 -16, año 2016 Facultad de Ingenierías y Arquitectura

Universidad de Pamplona

y contribuye a mejorar la producción de gas y en consecuencia la digestibilidad de este material lignificado, debido al mayor y mejor acceso a la celulosa por parte de los microorganismos ruminales (Akin et al., 1993; Kaur et al., 2012).

Finalmente parece ser que $P$. ostreatus tiene la capacidad de mejorar las características de la paja de arroz, incluso mejor que otros hongos de su mismo género (Pleurotus pulmonarius, Pleurotus tuber-reguim), como lo demuestran Akinfemi y Ogunwole (2012), quienes evaluaron a estos hongos en tamo de arroz y encontraron resultados similares al este estudio, como mayor producción de gas, micro minerales y PC, así como un menor contenido de FDN y LDA. Con lo cual se soporta la idea de que el tratamiento de este tipo de subproducto bajo la simplicidad de este método propuesto, es viable para ser utilizado en producción animal, especialmente en ganado rumiante.

\section{CONCLUSIÓN}

Hay un importante efecto sobre la calidad nutricional del tamo de arroz tratado con $P$. ostreatus, reflejada en el aumento de PC y producción de gas, así como en la reducción del contenido de lignina y FDN, que lo convierte en un alimento potencial para ganado bovino. Se abren interesantes áreas de investigación relacionada con el uso de enzimas para mejorar de la calidad nutricional de este subproducto de importancia regional y nacional.

\section{REFERENCIAS}

Abdel-Hamid, A.M., Solbiati, J.O., y Cann, I.K.O. (2013). Insights into Lignin Degradation and its Potential Industrial Applications. Advances in Applied Microbiology, 82,1-28.

Abril, D., Navarro. E., y Abril, A. (2009). La Paja de Arroz. Consecuencias de su Manejo y Alternativas de Aprovechamiento. Agron, 17(2), 69 - 79.
AOAC. (1990). Official methods of analysis of the association of oficial analytical chemists. $15^{\text {th }}$ ed. Arlington. Virginia. USA. Method. 942.05.

Akin, D.E., Sethuraman, A., Morrison, W.H., Martin, S.A., y Eriksson, K. E. (1993). Microbial delignification with White rot 
@ @IMENTECH CIENCIA Y TECNOLOGÍA ALIMENTARIA ISSN 1692-7125. Volumen 14 No. 1, p. 5 -16, año 2016 Facultad de Ingenierías y Arquitectura Universidad de Pamplona

fungi improves forage digestibility. Appl Environ Microbiol, 59, 4272 -4282.

Akinfemi, A., y Ogunwole, O. A. (2012). Chemical composition and in vitro digestibility of rice straw treated with Pleurotus ostreatus, Pleurotus pulmonarius and Pleurotus tuber-regium. Slovak J. Anim. Sci, 45(1), 14-20.

Barros, P.V. (2009). Evaluación de un subproducto de destilería de alcohol (vinaza) como aditivo en la alimentación de pollos de engorde (Tesis de pregrado). Universidad de Riobamba. Riobamba. Ecuador.

Cohen, R., Persky, L., y Hadar, Y. (2002). Biotechnological applications and potential of wood degrading mushrooms of the genus Pleurotus. Applied Microbiology and Biotechnology, 58, 582594.

Dairy N. Z, (2008). Pastures and Supplements for Grazing Animals NZSAP: Milk Production from Pasture Holmes. Occasional Publication, 14. Disponible en: http://www.dairynz.co.nz/media/253603/1 $=$

2 Principles of Grazing Management.p df. Consultado: 26/02/2016.

Diaz, C. C., Medina, A., Villamizar A., Palencia, D., Efecto de un suplemento

líquido a base de Saccharomyces cerevisiae y Lactobacillus casei para la alimentación de mojarra roja. (Oreochromis sp) en la etapa de alevinaje y precria, (2014). @limentech, Ciencia y Tecnología alimentaria. ISSN: 1692-7125. Vol. 12. N¹. P. 86 -92.

Drake, D.J., Nader, G., y Forero, L. (2002). Feeding Rice Straw to Cattle. University of California, 8079,1-18. Disponible en: http://anrcatalog.ucanr.edu/pdf/8079.pdf.

Consultado: 25/02/2016.

Eggen, T. (2000). Bioremediation of recalcitrant aromatic organic pollutants with white rot fungi (Doctor theses). Jordforsk, Norway. Agricultural University of Norway.

FAO. (2012). Crop residue based densified total mixed ration. A user-friendly approach to utilise food crop by-products for ruminant production, by T.K. Walli, M.R. Garg y Harinder P.S. Makkar. FAO Animal Production and Health Paper. 172. Rome, Italy.

Ferreira, L. R. (2009). Biodegradação de vinhaça proveniente do processo industrial de cana-de açúcaror fungos (Tese doutor). Universidade de São Paulo. São Paulo. Brasil.

France, J., Dhanoa, M.S., Theodorou, M.K., Lister, S.J., Davies, D.R., y Issac, D. A. 
@LIMENTECH CIENCIA Y TECNOLOGÍA ALIMENTARIA ISSN 1692-7125. Volumen 14 No. 1, p. 5 -16, año 2016 Facultad de Ingenierías y Arquitectura

Universidad de Pamplona

(1993). Model to interpret gas accumulation profiles associated with in vitro degradation of ruminant feeds. $J$ Theorical Biol, 163. 99-111.

González, A.M., Arbo. M. (2013). Botánica Morfológica Morfología de Plantas Vasculares Universidad Nacional del Nordeste Facultad de Ciencias Agrarias Argentina. Disponible en: www.biologia.edu.ar/botanica.

Consultado: 26/02/2016.

Hungate, R.E. (1988). Introduction: The ruminant and the rumen. In: Hobson, P.N. editor. The Rumen Microbial Ecosystem. Elsevier Applied Science. London and New York.

Higuchi, T. (2004). Microbial degradation of lignin: Role of lignin peroxidase, manganese peroxidase, and laccase. Proceedings of the Japan Academy, 80, 204-214.

Kaur, K., Wadhwa, M., Bakshi, M.P.S., y Kapoor, S. (2012). Nutritional evaluation of Pleurotus florida and Pleurotus sajorcaju harvested spent wheat-rice straw as livestock feed. Indian Journal of Animal Sciences, 82(9), 1033-1037.

Karunanandaa, K., Varga, G. A., Akin, D. E., Rigsby, L. L., y Royse, D. J. (1995). Botanical fractions of rice straw colonized by white-rot fungi: changes in chemical composition and structure. Animal Feed Science and Technology, 55(3), 179-199.

Menke, K. H., y Steingass, H. (1988). Estimation of the energetic feed value obtained from chemical analysis and in vitro gas production using rumen fluid. Animal Research and Development, 28, 7-55.

Montañez O. D., Ortega C. M., Cobos, P. M., Larque, A. y Garca, M. J. E. (2004). Efecto de la alimentacín con paja de trigo tratada con Pleurotus florida en la flora ruminal de ovinos. Rev. Cub. Cienc. Agrc, 38, 249257.

Moreira, S.L. (2006). Enzimas ligninolíticas produzidas por Psilocybecastanella CCB444 em solo contaminado com hexaclorobenzeno (Mestrado em Biodiversidade Vegetal e Meio Ambiente). Instituto de Botânica da Secretaria do Meio Ambiente. São Paulo. Brasil.

Mushrooms, F. O. E. (2007). Fortificación de hongos comestibles (Pleurotus ostreatus) con calcio, selenio y vitamina C. Vitae, 14(1), 16-24.

Peláez, A. A. (2010). Producción de enzimas lacasas e isoformas del gênero Pleurotus spp sobre bagazo de caña de azúcar por cultivo sólido (Tesis doctoral). Colegia de postgraduados Institución de enseñanza 
@LIMENTECH CIENCIA Y TECNOLOGÍA ALIMENTARIA ISSN 1692-7125. Volumen 14 No. 1, p. 5 -16, año 2016 Facultad de Ingenierías y Arquitectura

Universidad de Pamplona

e Investigación en ciencias agrícolas. Montecillo. México.

Pompeu, G.B. (2010). Comportamento enzimático de quatro fungos lignoceluloliticos crescidos em bagaço e palha de cana de açúcar e expostos a duas concentrações de nitrogênio, visando à produção de etanol (Tese doutorado). Centro de Energia Nuclear na Agricultura, Universidade de São Paulo, Piracicaba, Brasil.

Rangel, S. X. (2012). Estudio del efecto de enzimas ligninolíticas y celulolíticas obtenidas del hongo Pleurotus ostreatus sobre una gramínea forrajera tropical (Tesis de maestría) .Universidad Nacional de Colombia. Bogotá. Colombia.

Taniguchi, M., Suzuki, H., Watanabe, D., Sakai, K., Hoshino, K., y Tanaka, T. (2005). Evaluation of Pretreatment with Pleurotus ostreatus for Enzymatic Hydrolysis of Rice Straw. Journal of Bioscience and Bioengineering 100(6), 637-643.

Van Soest. P.J. (2006). Rice straw, the role of silica and treatments to improve quality. Animal Feed Science and Technology, 130, 137-171. 